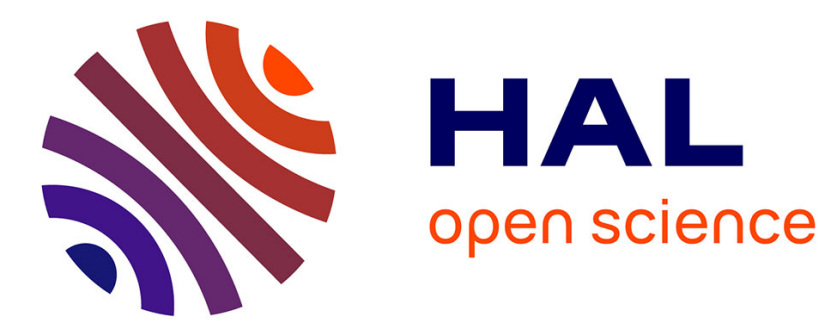

\title{
Discrete surfaces segmentation into discrete planes
}

Isabelle Sivignon, Florent Dupont, Jean-Marc Chassery

\section{To cite this version:}

Isabelle Sivignon, Florent Dupont, Jean-Marc Chassery. Discrete surfaces segmentation into discrete planes. International Workshop on Combinatorial Image Analysis, 2004, New Zealand. pp.458-473. hal-00185217

\section{HAL Id: hal-00185217 https://hal.science/hal-00185217}

Submitted on 6 Nov 2007

HAL is a multi-disciplinary open access archive for the deposit and dissemination of scientific research documents, whether they are published or not. The documents may come from teaching and research institutions in France or abroad, or from public or private research centers.
L'archive ouverte pluridisciplinaire HAL, est destinée au dépôt et à la diffusion de documents scientifiques de niveau recherche, publiés ou non, émanant des établissements d'enseignement et de recherche français ou étrangers, des laboratoires publics ou privés. 


\title{
Discrete Surfaces Segmentation into Discrete Planes
}

\author{
Isabelle Sivignon $^{1}$, Florent Dupont $^{2}$, and Jean-Marc Chassery ${ }^{1}$ \\ 1 Laboratoire LIS - Institut National Polytechnique de Grenoble, \\ 961, rue de la Houille Blanche - BP46, \\ 38402 St Martin d'Hères Cedex \\ \{sivignon, chassery\}@lis.inpg.fr \\ 2 Laboratoire LIRIS - Université Claude Bernard Lyon 1, \\ Bâtiment Nautibus - 8, boulevard Niels Bohr, \\ 69622 Villeurbanne Cedex \\ fdupont@liris.cnrs.fr
}

\begin{abstract}
This paper is composed of two parts. In the first one, we present an analysis of existing discrete surface segmentation algorithms. We show that two classes of algorithms can actually be defined according to discrete surface and plane definitions. In the second part, we prove the link between the two classes presented. To do so, we propose a new labelling of the surface elements which leads to a segmentation algorithm of the first class that can be easily transformed into a segmentation algorithm of the second class.
\end{abstract}

\section{Introduction}

The segmentation of a discrete object surface into pieces of discrete planes is the first step of more global processes like polyhedrization of the surface 1 or surface area estimation 2. This problem can be stated as follows: given a discrete object, a pair of connectivities used for the object and the background, and a definition of the discrete surface, label all the surface elements of the object such that the elements with the same label belong to the same piece of discrete plane. Each set of surface elements with the same label is called a discrete face.

This statement raises the problem that both discrete surfaces and discrete planes have to be defined before the segmentation process. In a general way, there exist two families of discrete surfaces definition: either the voxels or the surfels are the surface elements. In the same way, two types of discrete planes are mainly used: the naive planes and the standard planes.

In the literature, those discrete surface and discrete plane definitions are all used in segmentation algorithms, but most of the time, few arguments are given to justify the use of one definition instead of another. Some authors even propose several algorithms using different planes and surfaces definitions, but did not explore the relation between the different algorithms [3]. Indeed, by now, no work has been accomplished in order to study the link between, first, the discrete 
surface and discrete plane definitions, and second, the segmentation processes that use different kinds of discrete planes.

In Section 2, we define those discrete surfaces and point out the links between the surfaces and the different types of discrete planes. Then, we propose a short overview of existing segmentation algorithms, in relation with the surface and plane definitions given previously. Next, in Section 3, we recall a segmentation algorithm we proposed in a previous work. This algorithm uses naive planes over the surface voxels and is quite different from existing algorithms since the voxels' faces are labelled instead of the voxels themselves. We moreover show how the 6 connectivity between the object voxels can be handled in this algorithm. Finally, in Section 4, we propose a transformation from this segmentation algorithm with naive planes into a segmentation algorithm with standard planes. This transformation shows that the two segmentation processes are in fact equivalent. Some image results are given before a few words of conclusion.

\section{Different Discrete Planes for Different Kind of Surfaces}

\subsection{Two Types of Surfaces}

The two existing approaches differ by the surface elements considered: the surface elements are either voxels (dimension 3 ) or voxels' faces (dimension 2).

In his $\mathrm{PhD}$ thesis, Perroton [4] calls those approaches homogeneous and heterogeneous respectively, since in the first one the surface elements are space elements, whereas in the second one the dimension of the surface elements is lower than the dimension of the discrete space.

Homogeneous Approach. In 1982, a definition of discrete surface in dimension $n$ is proposed by Udupa et al. [5], and a surface tracking algorithm is described. Nevertheless, no Jordan's theorem proving that this surface separates the object voxels from the background voxels has been shown with this definition. The year before, Morgenthaler et al. 6] gave a local definition of a discrete surface, and a global Jordan's theorem based on this definition is shown. Malgouyres also proposed 7] a local definition for 26-connected discrete objects with a 6connected background. A Jordan's theorem is also shown and the link between Morgenthaler's surfaces is stated [8,7]. Another surface definition involving $n$ dimensional surface elements has been recently proposed in 9, where separating pairs of connectivity are studied according to this definition.

Nevertheless, this approach causes some problems [4]: first, the surface of an object and the surface of the background are different, and second, for some objects containing a cavity, the two surfaces expected (two surfaces should separate the object from the two background components: the cavity, and the infinite background) may have voxels in common.

Heterogeneous Approach. This approach is based on the abstract cellular complex introduced by Kovalevsky [10] for discrete images topology. In dimension 3, the following cells are defined (see Figure 1(a)): 
- voxel: cell of dimension 3

- surfel: cell of dimension 2

- linel: cell of dimension 1

- pointel: cell of dimension 0 .

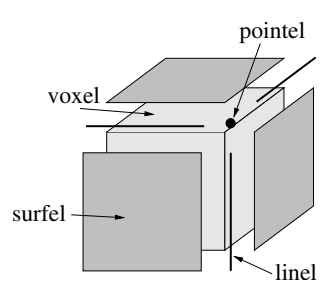

(a)

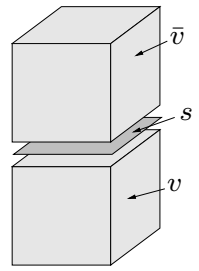

(b)

Fig. 1. (a) Cellular decomposition of a voxel. (b) A surfel defined by two 6-connected voxels

Surfels can also be defined as pairs of voxels:

Definition 1. A surfel is the common face of a couple of 6-connected voxels. We denote $s=\{v, \bar{v}\}$ the surfel defined by the two 6-connected voxels $v$ and $\bar{v}$.

The surface of an object is directly derived from this definition:

Definition 2. Let $\mathcal{O}$ be a $3 D$ discrete object. The surface of $\mathcal{O}$ is defined as the set of surfels $s=\{v, \bar{v}\}$ such that $v \in \mathcal{O}$ and $\bar{v} \notin \mathcal{O}$.

In 1992, Herman [1] generalizes this definition to any dimension. This definition has moreover been used to write surface tracking algorithms: first, Artzy et al. 12 proposed a tracking algorithm for 18-connected objects; then, Gordon and Udupa 13 extend this algorithm for 6-connected objects improving it; in 1994, Udupa [14] adds a connectivity definition over surfels, and proposes a very efficient tracking algorithm; in [15], Perroton describes a parallel surface tracking algorithm for 26-connected objects.

In the following, we will see how this definition may be used in two different ways in the context of surface segmentation.

\subsection{Which Planes for Which Surfaces?}

In a segmentation process, the discrete points of the object surface are labelled according to the discrete face they belong to. In the literature, two main approaches have been developed: the discrete points are either the voxels or the pointels of the object.

The definition of a discrete surface given in Definition 2 states that the surface is composed of all the surfels $s=\{v, \bar{v}\}$ such that $v \in \mathcal{O}$ and $\bar{v} \notin \mathcal{O}$. From this definition, we derive that the segmentation is done over the pointels of the object surface. Nevertheless, another point of view is given by the definition of the inner boundary of an object: 
Definition 3. Let $\mathcal{O}$ be a $3 D$ discrete object. The inner boundary of $\mathcal{O}$ is defined as:

$$
I I(\mathcal{O})=\{v \mid\{v, \bar{v}\} \text { is a surfel of } \mathcal{O} \text { 's surface }\}
$$

The voxels of $I I(\mathcal{O})$ are the underlying voxels of the surface surfels. When a segmentation is done over the object voxels, the voxels of $I I(\mathcal{O})$ are usually labelled.

Let us recall the analytical definition of a discrete plane [16, 17]:

Definition 4. A discrete plane of parameters $(a, b, c, \mu)(a, b, c$ and $\mu$ belong to $\mathbb{Z}$ and $a, b$ and $c$ are relatively prime) and thickness $\omega$ is the set of voxels $(x, y, z)$ verifying the following inequalities:

$$
0 \leq a x+b y+c z+\mu<\omega
$$

The thickness parameter $\omega$ defines many plane classes, but two types of discrete planes are mainly used in the literature. When $\omega=\max (|a|,|b|,|c|)$, the discrete plane is called naive, and when $\omega=|a|+|b|+|c|$, the plane is called standard. Naive planes are the thinnest planes without 6 -connected holes, and standard planes are the thinnest planes without tunnels [17]. For further information about digital planarity, see for instance [18.

Table 1 presents the links between discrete planes and discrete surfaces. On one hand, if the discrete points are the voxels of the inner boundary, then naive planes are used for the segmentation. Indeed, the voxels that are 18-connected but not 6-connected with the background are needed for a segmentation with standard planes, but do not belong to the inner boundary of the object. On the first line of the table, a naive plane recognition has been done over the inner boundary of the object: the voxels of $I I(\mathcal{O})$ are labelled.

On the other hand, the pointels belonging to the object's surface are 6connected, which suits well to a segmentation with standard planes. On the second line of the table, a standard plane recognition has been done over the pointels of the surfel boundary of the object: a surfel is colored if and only if its four adjacent pointels belong to the discrete face.

\subsection{Short Overview of Existing Algorithms}

The first segmentation algorithm was proposed by Debled-Rennesson [19. She applies the naive plane recognition algorithm she proposed on the inner boundary of a discrete object. However, her algorithm works only for objects having known symmetries. With a totally different idea, Vittone proposes in [20] a segmentation algorithm based on the fact that any naive plane can be decomposed into basic structures [21. But only a few preliminary results are given and this method has not been further extended by now. The following year, Vittone 22] wrote another segmentation algorithm using the naive plane recognition algorithm she proposed. This recognition algorithm is based on a dual transformation, which we also use in our work. This will be detailed in the next section. In [3], Papier proposes another segmentation algorithm based on Debled-Rennesson naive 
Table 1. Links between the discrete surfaces and the discrete planes used for the segmentation process

\begin{tabular}{|c|c|c|c|}
\hline Surface definition & $\begin{array}{c}\text { Discrete } \\
\text { points }\end{array}$ & Discrete planes \\
\hline Inner boundary & Voxels & Naive planes \\
\hline Set of surfels & Pointels & Standard planes & \\
\hline & &
\end{tabular}

plane recognition algorithm. However, his algorithm does not give satisfactory results since many small discrete faces (with a few voxels) are recognized.

This observation led him to propose a segmentation algorithm of the surfel boundary with standard planes [1. The discrete faces recognized are forced to be connected and homeomorphic to disks in order to be used in a polyhedrization process. A Fourier-Motskin elimination algorithm is used to recognize the standard planes, which makes the overall algorithm complexity high. Moreover, many small discrete faces are still recognized by this algorithm, and some tricks like discrete face size limitations are proposed to better the results. Finally, Klette and Sun proposed in 2] a segmentation algorithm close to the one of Papier in order to estimate the object surface area. In this case, no particular constraint over the shape of the discrete faces is required, except the connectivity, which is implicitly obtained since a breadth-first tracking of the surfels is done.

For the algorithms of the first paragraph, a segmentation of the inner boundary is done, and the voxels are labelled. The algorithms of the second paragraph use a standard plane segmentation of the object pointels, and a surface surfel is labelled by a discrete face when its four adjacent pointels belong to the face.

\section{$3 \quad$ Surfel Labelling Using Naive Planes}

In this section, we propose a segmentation algorithm of the inner boundary of an object using naive planes. But instead of labelling the voxels of the inner boundary, we label only some of the boundary's surfels. Indeed, all the existing algorithms using naive planes label the voxels of the object inner boundary. Nevertheless, the inner boundary definition is derived from the surfel surface def- 
inition. Thus the surface elements are the surfels, and it seems more appropriate to label the surfels. In Section 4, we show how this segmentation of the inner boundary can be easily transformed into a segmentation into standard planes, which link the two segmentation approaches existing by now.

Since the final segmentation uses standard planes, we consider a 6connectivity relationship for the voxels of the object. Indeed, 18- and 26- connectivities do not enable the description of the object surfel surface as a 2dimensional combinatorial manifold, which is inconsistent with the use of standard planes 23 .

\subsection{Algorithm Description}

Part of this algorithm is presented in [24] and we first recall here the main points of this method.

The general idea of this algorithm is to get the following labelling of the surface surfels: if a surfel $s=\{v, \bar{v}\}$ of the surface ( $v$ belongs to the object and $\bar{v}$ does not) is labelled with the discrete face $f$, then there exists a Euclidean plane $p$ crossing the segment $[v \bar{v}$. In other words, the discretization of the plane $p$ with the OBQ discretization [25] scheme contains the voxels of $f$.

In the following, we denote by $l$ one direction of the set $\{(1,0,0),(-1,0,0)$, $(0,1,0),(0,-1,0),(0,0,1),(0,0,-1)\}$. Then for each voxel $v$ of the inner boundary of an object, there exist at least one direction $l$ such that the voxel $v+l$ does not belong to the object. The naive plane recognition used is a directional recognition algorithm:

Definition 5. Let $V$ be a set of voxels. The directional recognition in direction $l$ computes the set of Euclidean planes crossing all the segments $[v \bar{v}[$ such that $v \in V$ and $\bar{v}=v+l$.

This recognition is achieved using Vittone's naive plane recognition algorithm 22. This algorithm is based on a dual transformation. Consider for instance the direction $l=(0,0,1)$. Then the Euclidean planes of parameters $\left(\alpha_{0}, \beta_{0}, \gamma_{0}\right)$ which cross the segment $[v, v+l[$ where $v=(x, y, z)$ are those fulfilling the double inequality $0 \leq \alpha_{0} x+\beta_{0} y+z+\gamma_{0}<1$. Then, in the parameter space $(\alpha, \beta, \gamma)$, the set of Euclidean planes computed by the directional algorithm on a set $V$ is a convex polyhedron defined as the intersection of all the double inequalities related to the voxels of $V$.

But this recognition algorithm enables to label only the surfels $s=\left\{v, v^{\prime}\right\}$ where $v^{\prime}=v+l$ if $l$ is the direction of recognition (see Figure 2(a)). A second step of the algorithm is to label the inner surfels of the plane, which are the surfels sharing two edges with surfels already labelled by the directional algorithm (see Figure 2(b) and (c)). It is easy to prove that the Euclidean planes that cut the segments related to the two surfels defining an inner surfel also cut the segment related to this inner surfel.

After this short presentation of the recognition process used, let us see how this algorithm is applied on a surface. Since the quality of the segmentation is not the main point of this paper, and for the sake of clarity of the rest of this 


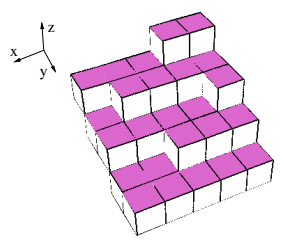

(a)

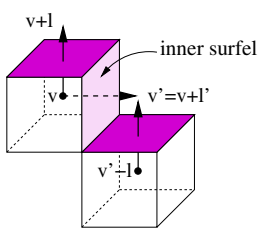

(b)

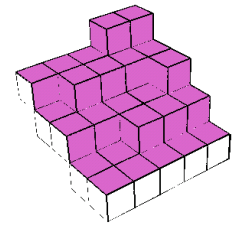

(c)

Fig. 2. Labelling of the surfels of a piece of plane of parameters $(1,3,-5,0)$ using the directional algorithm in direction $(0,0,1)$ (from [24]): (a) just after the directional algorithm; (b) illustration of an inner surfel; (c) after the labelling of inner surfels

work, we do not present the algorithm described in [24, but quickly explain a simple method. A seed voxel $v_{0}$ is chosen for the discrete face to recognize in direction $l$. Neighbour voxels are added one by one if they belong to the same naive plane as the voxels of $f$ and if they fulfill the condition presented in the next paragraph. This process starts over with a new seed voxel until all the surfels are labelled.

\section{$3.2 \quad 6$-Connected Objects}

Since 6-connectivity is considered for the discrete object, a discrete face recognized on the object surface should not be split over two different 6-connected components. Since naive planes are 18-connected, we have to define the cases where two voxels are 18-connected in a 6 -connected object.

Definition 6. Let $v$ and $v^{\prime}$ be two voxels of a 6 -connected object $\mathcal{O}$. Then $v$ and $v^{\prime}$ are 18-neighbours in $\mathcal{O}$ if and only if one of the following two conditions is fulfilled (see Figure 3 ):

- $v$ and $v^{\prime}$ are 6-connected (they share a surfel)

- $v$ and $v^{\prime}$ share a linel, and one out of the other two voxels sharing this linel belongs to $\mathcal{O}$.

Now consider a directional recognition in direction $l$.

Definition 7. Two voxels $v$ and $v^{\prime}$ are l-neighbours in a 6 -connected object $\mathcal{O}$ if only if they fulfill the following three conditions:

- $v$ and $v^{\prime}$ belong to the inner boundary of $\mathcal{O}$ and the voxels $v+l$ and $v^{\prime}+l$ do not belong to $\mathcal{O}$

- the projection of $v$ and $v^{\prime}$ along direction $l$ are 4-connected

- $v$ and $v^{\prime}$ are 18-neighbours in $\mathcal{O}$ (see Definition 6).

Figure 4 presents some examples of voxels that are or are not $l$-neighbours. Finally, during the segmentation process, a new voxel can be added to a discrete face $f$ recognized in direction $l$ if and only if it is $l$-neighbour with a voxel of $f$. 


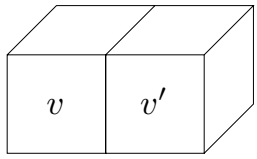

(a)

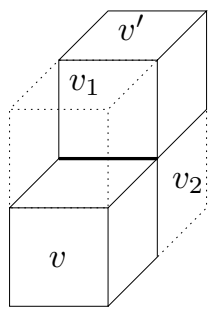

(b)

Fig. 3. The voxels $v$ and $v^{\prime}$ are 18-connected in a 6-connected object if and only if they are in configuration (a) or if $v_{1}$ or $v_{2}$ belongs to the object in configuration (b)

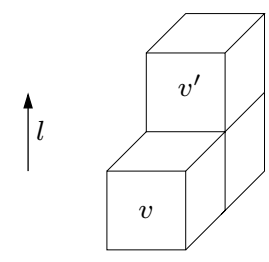

(a)

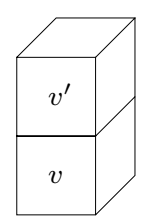

(b)

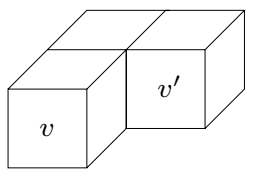

(c)

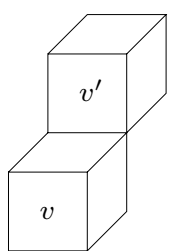

(d)

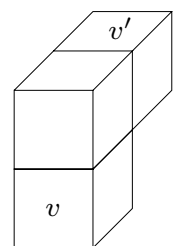

(e)

Fig. 4. The two voxels $v$ and $v^{\prime}$ are $l$-neighbours (see $l$ on the left) only in configuration (a) : in (b), the first condition of Definition 7 is not fulfilled since the surfel $\{v, v+l\}$ does not belong to the surface; in (c), the second condition is not fulfilled since the projections of $v$ and $v^{\prime}$ along direction $l$ are not 4-connected; in (d), the two voxels dot not fulfill the conditions of Definition 6; in (e), the two last conditions are fulfilled, but like in (b), the first one is not

Examples of segmentation results using 6-connectivity are proposed in Figure 5. The recognition in direction $(0,0,1)$ only has been computed on those examples. In (a), one unique plane is recognized; in (b), two planes are recognized since the object is composed of two 6-connected components; in (c), three planes are recognized, one for each 6-connected component.

\subsection{Results}

Some results of the algorithm proposed in [24] with the 6-connectivity constraint are presented in Figure 6. The results obtained with the simple algorithm presented in this paper simply contain more small faces. The first image (a) is an ellipsoid of parameters $(20,16,10)$ which is composed of 4190 surface surfels. 131 discrete faces are recognized on this surface, and 15 among them are composed of less than 5 surfels. The image in (b) is a microscopic scale view of part of a bone. Its surface is composed of 5298 surfels, and 201 discrete faces are recognized by the algorithm. 65 discrete faces are composed of less than 5 surfels. 


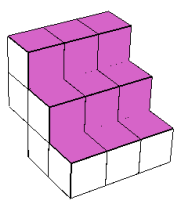

(a)

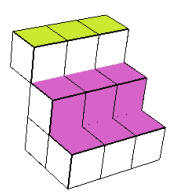

(b)

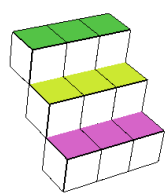

(c)

Fig. 5. Influence of the 6-connectivity on the segmentation computed

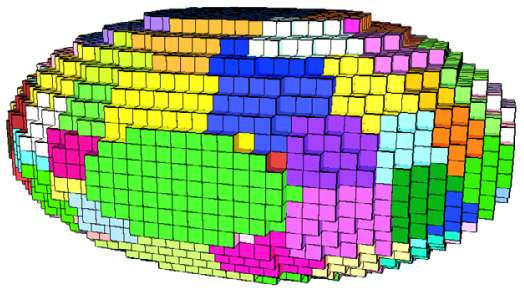

(a)

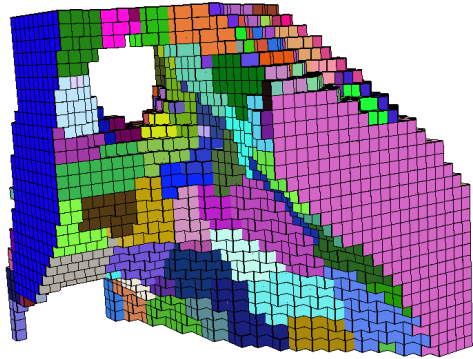

(b)

Fig. 6. Results of the segmentation algorithm of the inner boundary with naive planes: (a) an ellipsoid of parameters $(20,16,12)$ and (b) a piece of bone

\section{Segmentation Using Standard Planes}

In this section, we show that the segmentation using naive planes over the inner boundary voxels presented in the previous section can be very easily transformed into a segmentation of the surface pointels into standard planes.

\subsection{Transformation}

Let us consider an infinite 3D discrete object defined as a half-space bounded by a naive plane of parameters $(a, b, c, \mu)$. Let us moreover suppose that the normal vector of this plane is directed towards the discrete object itself. Then we study the surfel surface of this object (see Figure 7(a)).

We use here the definition of standard plane proposed in 26] and directly derived from the standard digitization scheme:

Definition 8. Let $P$ be a Euclidean plane defined by the equation $a x+b y+c z+$ $\mu=0$, with $a, b, c$ and $\mu$ integers. Then the standard digitization of $P$ is the set of voxels $(x, y, z)$ such that:

$$
-\frac{|a|+|b|+|c|}{2} \leq a x+b y+c z+\mu<\frac{|a|+|b|+|c|}{2}
$$




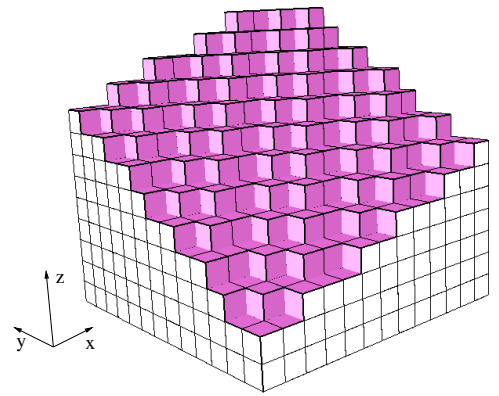

(a)

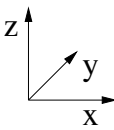

(b)

Fig. 7. (a) Discrete half-space bounded by the naive plane of parameters $(2,3,-5)$. The surfels of the surface are in grey. (b) Illustration of the bijective transformation between a voxel $V$ and a pointel $p$ for given plane parameters

if $(a>0)$ or $(a=0$ and $b>0$ or $(a=0$ and $b=0$ and $c>0)$ (called standard orientation). Otherwise, the left inequality is strict whereas the right one is not.

Note that the possible values of $a x+b y+c z+\mu$ are centered around zero. The following theorem makes the link between the naive plane parameters and the pointels of the surface.

Theorem 1. Let $P$ be a naive plane of parameters $(a, b, c, \mu)$ and $\mathcal{S}$ be the surfel surface described as above. Then the pointels belonging to $\mathcal{S}$ belong to the standard plane of parameters $\left(a, b, c, \mu^{\prime}\right)$ in the discrete grid translated by $t=\left(\frac{1}{2}, \frac{1}{2}, \frac{1}{2}\right)$, with:

$$
\mu^{\prime}=\tau-\frac{|a|+|b|+|c|}{2}-v \cdot(a, b, c)
$$

where

- $\tau=\left\{\begin{array}{r}\mu \text { if }(a>0) \text { or }(a=0 \text { and } b>0) \text { or }(a=0 \text { and } b=0 \text { and } c>0) \\ \mu+1 \text { otherwise }\end{array}\right.$

- $v=-\frac{1}{2}(\operatorname{sgn}(a), \operatorname{sgn}(b), \operatorname{sgn}(c))+\left(\frac{1}{2}, \frac{1}{2}, \frac{1}{2}\right)$

$(\operatorname{sgn}(x)$ is equal to 1 if $x>0,-1$ if $x<0$, and 1 or -1 if $x=0)$.

Proof. This proof works by construction. Figure 8 illustrates the different steps of this construction from a naive $2 \mathrm{D}$ segment to a standard $2 \mathrm{D}$ segment. This example will be detailed in the next paragraph.

Since we transform a naive plane into a standard plane, the first thing is to thicken the naive plane $P$. This is equivalent to adding some voxels that fulfill larger inequalities (the thickness determines the bounds of the inequalities in a discrete plane definition). This is done in three steps: 
- Since the inequalities defining a standard plane depend on the signs of the parameters, a preprocessing step is required when $(a<0)$ or $(a=0$ and $b<0)$ or $(a=0$ and $b=0$ and $c<0)$. We use the fact that the two doubleinequalities $0 \leq a x+b y+c z+\mu<\omega$ and $0<a x+b y+c z+\mu+1 \leq \omega$ are equivalent when integer values are considered to do the parameters translation $(a, b, c, \mu) \rightarrow(a, b, c, \mu+1)$ in the case of a non standard orientation. This operation simply swap the large and strict inequalities in the discrete plane definition. In the following, we suppose that the parameters are in a standard orientation since the two cases are similar.

- The thickness of a standard plane is $|a|+|b|+|c|$. Since we supposed that the normal vector is directed towards the object, the voxels $V=(x, y, z)$ fulfilling the condition $\max (|a|,|b|,|c|) \leq a x+b y+c z+\mu<|a|+|b|+|c|$ must be added to thicken the plane inside the discrete object.

- At this point, our plane is defined by the double inequality $0 \leq a x+b y+c z+$ $\mu<|a|+|b|+|c|$. To be consistent with Definition 8, a last translation of the parameters of vector $\left(0,0,0,-\frac{|a|+|b|+|c|}{2}\right)$ must be done.

All the voxels of the discrete object that are 18-connected or 26-connected with the background are added by those transformations, and the translation $(a, b, c, \mu) \rightarrow\left(a, b, c, \mu(+1)-\frac{|a|+|b|+|c|}{2}\right)$ has been done. The next part of the transformation consists in moving the discrete grid such that the discrete points are now the pointels.

A one-to-one and onto transformation is defined between the voxels of the plane $P$ after thickening and the pointels of the surface: the pointel $p=(x-$ $\left.\frac{1}{2} \operatorname{sgn}(a), y-\frac{1}{2} \operatorname{sgn}(b), z-\frac{1}{2} \operatorname{sgn}(c)\right)$ is associated to the voxel $V=(x, y, z)$. This transformation is of course bijective but we must prove that if $V$ belongs to $P$, then $p$ belongs to the surface of $\mathcal{O}$. Let us consider a plane of parameters $(a, b, c)$ with $a>0, b>0$ and $c<0$. The other cases are symmetrical. Then the transformation maps the point $V$ to the pointel $p=\left(x-\frac{1}{2}, y-\frac{1}{2}, z+\frac{1}{2}\right)$ (see Figure $7(\mathrm{~b})$ ). Only three types of voxels may appear in the thickened discrete plane $P$ :

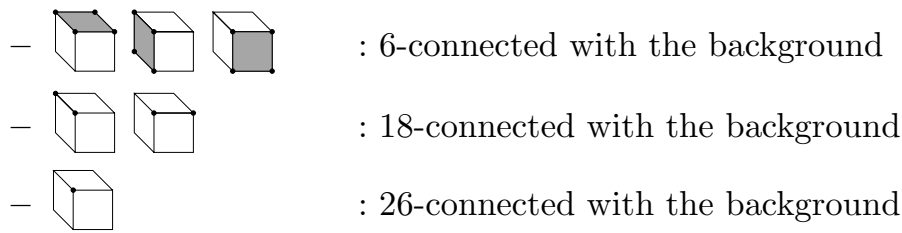

For each case, the pointels that belong to the surface are underlined. We note that for each voxel $(x, y, z)$ of the thickened plane $P$, the pointel $\left(x-\frac{1}{2}, y-\right.$ $\frac{1}{2}, z+\frac{1}{2}$ ) always belongs to $\mathcal{O}$ 's surface. For other parameters' signs a similar construction can be done, and finally, the transformation always maps a voxel of $P$ into a pointel of the surface. If one of the parameters $a, b$ or $c$ is equal to zero, then two pointels of each voxel of $P$ are always on $\mathcal{O}$ 's surface. Then two bijective transformations are possible and correspond to the two possible values of $\operatorname{sgn}(x)$ when $x=0$. When two out of the three parameters are equal to zero, then 4 
pointels of each voxel belong to $\mathcal{O}$ 's surface, and then four transformations are possible.

This transformation defines the first part of the translation vector $v$ of the theorem, and a simple translation of the grid of the vector $t=\left(\frac{1}{2}, \frac{1}{2}, \frac{1}{2}\right)$ leads to the final result.

The following Corollary shows how to transform a segmentation with naive planes into a segmentation with standard planes:

Corollary 1. Let $f$ be a discrete face recognized on the inner boundary of a discrete object $\mathcal{O}$ with the algorithm presented in Section 3. If the OBQ discretization of the Euclidean plane of parameters $(a, b, c, \mu)$ contains the voxels of $f$, then the standard discretization of the plane $\left(a, b, c, \mu^{\prime}\right)$ (where $\mu^{\prime}$ is defined as in Theorem 1) contains all the pointels belonging to the labelled surfels of $f$, in a discrete grid translated by $t=\left(\frac{1}{2}, \frac{1}{2}, \frac{1}{2}\right)$.

The proof of this corollary is straightforward from Theorem 1,

\subsection{Example}

Figure 8 is an example of the transformation steps of Theorem 1 for a discrete segment. The first column represents the discrete segment, the second one represents the set of Euclidean solution lines in the parameter space, and the third one the parameters of the Euclidean lines related to the vertices of the convex polygon of the parameter space.

On the first line, a segment of the naive line of parameters $(2,-3,3)$ is represented. On the second line, the naive segment has been thickened in order to get a standard segment. The parameters translation corresponding to this thickening is done. The third line represents the standard pointel segment derived from the standard voxel segment of the second line. A second translation of the Euclidean planes parameters is accomplished.

\subsection{Results}

Since the transformation presented above only concerns the parameters of the Euclidean planes recognized, visual results are hard to show. Nevertheless, Figure 9 presents a result of this transformation for a catenoid 1 . In (a), the surfel labelling using a naive plane segmentation over the inner boundary is represented: all the voxels containing surfels of the same color belong to the same discrete naive plane. In (b), the pointel labelling is represented after the transformation defined in Theorem 1; all the pointels with the same color belong to the same standard discrete plane. The same colors are used for the two images, so that the comparison is easier. Note that since each pointel belongs to several discrete faces, its color is the one of the first recognized face it belongs to.

${ }^{1}$ This image has been generated using the volgen program available on http://www.cb.uu.se/tc18/code_data_set/Code/Volgen/ 
Discrete segment
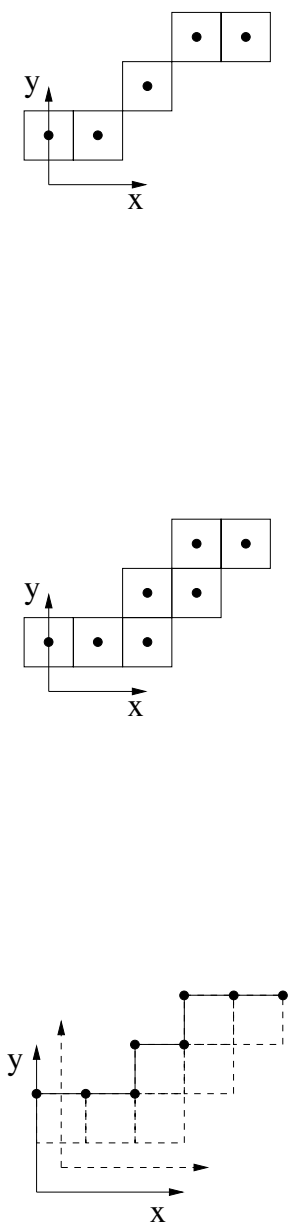

Solution Euclidean planes in the parameter space $(\alpha, \beta)$

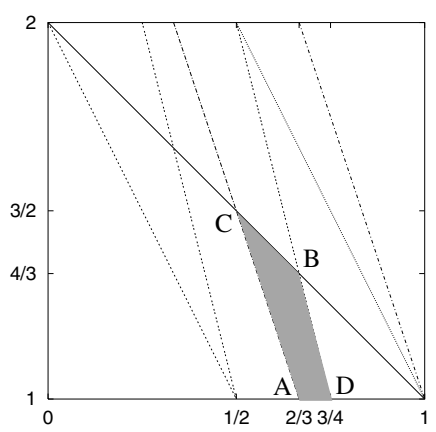

- $A=(2,-3,3)$

- $B=(2,-3,4)$

- $C=(1,-2,3)$

- $D=(3,-4,4)$

- $A=\left(2,-3, \frac{1}{2}\right)$

- $B=\left(2,-3, \frac{3}{2}\right)$

- $C=\left(1,-2, \frac{3}{2}\right)$

- $D=\left(3,-4, \frac{1}{2}\right)$

$$
\downarrow \mu-b
$$

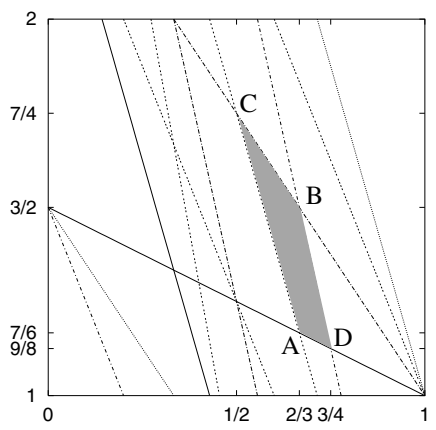

- $A=\left(2,-3, \frac{7}{2}\right)$

- $B=\left(2,-3, \frac{9}{2}\right)$

- $C=\left(1,-2, \frac{7}{2}\right)$

- $D=\left(3,-4, \frac{9}{2}\right)$

Fig. 8. All the steps from a naive voxel segment to a standard pointel segment

\section{Conclusion}

In this paper, we showed that two classes of discrete surface segmentation algorithms can actually be considered: the first one consists in looking for naive planes over the surface inner boundary, and the second one uses standard planes 


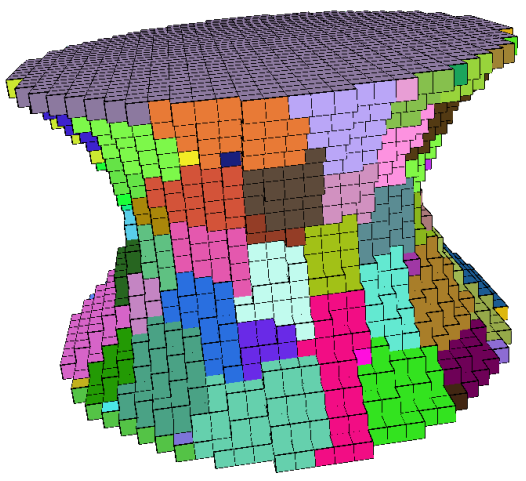

(a)

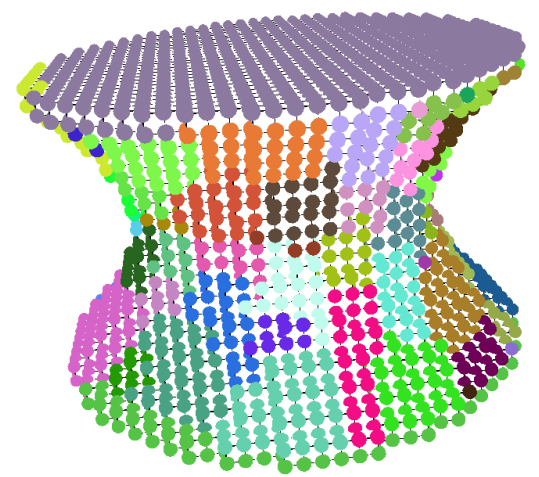

(b)

Fig. 9. Segmentation of a discrete catenoid surface: (a) surfel labelling; (b) pointel labelling

over the surface surfels. We recalled the different existing segmentation algorithms highlighting the fact that they all belong to one of those two classes.

The second part of this work was to show that those two types of segmentation are equivalent, and we expressed the transformation that changes from one to the other. To do this study, we proposed a segmentation algorithm using naive planes and generating a surfel labelling for 6-connected discrete objects.

Since the two segmentations are equivalent, an interesting future work would be to propose a complete study on the quality of segmentation algorithms, pointing out the influence of the choice of the first voxels of the discrete faces for instance. Such a study is very useful since the quality of the surface segmentation induces part of the quality of the polyhedrization or surface area estimation based on it.

\section{References}

1. Françon, J., Papier, L.: Polyhedrization of the boundary of a voxel object. In Bertrand, G., Couprie, M., Perroton, L., eds.: Discrete Geometry for Computer Imagery. Volume 1568 of Lect. Notes on Comp. Sci., Marne-la-Vallée, France, Springer-Verlag (1999) 425-434

2. Klette, R., Sun, H.J.: Digital planar segment based polyhedrization for surface area estimation. In Arcelli, C., Cordella, L.P., Sanniti di Baja, G., eds.: International Workshop on Visual Form. Volume 2059 of Lect. Notes on Comp. Sci., Capri, Italie, Springer-Verlag (2001) 356-366

3. Papier, L.: Polyédrisation et visualisation d'objets discrets tridimensionnels. PhD thesis, Université Louis Pasteur, Strasbourg, France (1999)

4. Perroton, L.: Segmentation parallèle d'images volumiques. $\mathrm{PhD}$ thesis, Ecole Normale Supérieure de Lyon, Lyon, France (1994) 
5. Udupa, J.K., Srihari, S.N., Herman, G.T.: Boundary detection in multidimension. IEEE Trans. on Pattern Anal. and Mach. Intell. 4 (1982) 41-50

6. Morgenthaler, D.G., Rosenfeld, A.: Surfaces in three-dimensional digital images. Information and Control $\mathbf{5 1}$ (1981) 227-247

7. Malgouyres, R.: A new definition of surfaces of $\mathbb{Z}^{3}$. A new 3D Jordan theorem. Theoretical Computer Science 186 (1997) 1-41

8. Bertrand, G., Malgouyres, R.: Some topological properties of discrete surfaces. In Miguet, S., Montanvert, A., Ubéda, S., eds.: Discrete Geometry for Computer Imagery. Volume 1176 of Lect. Notes on Comp. Sci., Lyon, France, Springer-Verlag (1996) 325-336

9. Brimkov, V., Klette, R.: Curves, surfaces and good pairs. Technical Report CITRTR-144, CITR, Auckland (NZ) (2004)

10. Kovalevsky, V.: Finite topology as applied to image analysis. Computer Vision, Graphics and Image Processing 46 (1989) 141-161

11. Herman, G.T.: Discrete multidimenional jordan surfaces. Computer Vision, Graphics and Image Processing 54 (1992) 507-515

12. Artzy, E., Frieder, G., Hreman, G.T.: The theory, design, implementation and evaluation of a three-dimensional surface detection algorithm. Computer Graphics and Image Processing 15 (1981) 1-24

13. Gordon, D., Udupa, J.K.: Fast surface tracking in three-dimensional binary images. Computer Vision, Graphics and Image Processing 45 (1989) 196-214

14. Udupa, J.K.: Multidimensional digital boundaries. Computer Vision, Graphics and Image Processing 56 (1994) 311-323

15. Perroton, L.: A new 26-connected objects surface tracking algorithm and its related pram version. Journal of Pattern Recognition and Artificial Intelligence 9 (1995) 719-734

16. Réveillès, J.P.: Géométrie discrète, calcul en nombres entiers et algorithmique. Thèse d'etat, Université Louis Pasteur, Strasbourg, France (1991)

17. Andrès, E., Acharya, R., Sibata, C.: Discrete analytical hyperplanes. Graphical Models and Image Processing 59 (1997) 302-309

18. Brimkov, V., Coeurjolly, D., Klette, R.: Digital Planarity - A Review. Technical Report CITR-TR-142, CITR, Auckland (NZ) (2004)

19. Debled-Rennesson, I.: Etude et reconnaissance des droites et plans discrets. PhD thesis, Université Louis Pasteur, Strasbourg, France (1995)

20. Vittone, J.: Caractérisation et reconnaissance de droites et de plans en géométrie discrète. PhD thesis, Université Joseph Fourier, Grenoble, France (1999)

21. Vittone, J., Chassery, J.M.: $(n-m)$-cubes and farey nets for naive planes understanding. In Bertrand, G., Couprie, M., Perroton, L., eds.: Discrete Geometry for Computer Imagery. Volume 1568 of Lect. Notes on Comp. Sci., Marne-la-Vallée, France, Springer-Verlag (1999) 76-87

22. Vittone, J., Chassery, J.M.: Recognition of digital naive planes and polyhedrization. In: Discrete Geometry for Computer Imagery. Volume 1953 of Lect. Notes on Comp. Sci., Springer-Verlag (2000) 296-307

23. Françon, J.: Sur la topologie d'un plan arithmétique. Theoretical Computer Science 156 (1996) 159-176

24. Coeurjolly, D., Guillaume, A., Sivignon, I.: Reversible discrete volume polyhedrization using marching cubes simplification. In Latecki, L.J., Mount, D.M., Wu, A.Y., eds.: Vision Geometry XII. Volume 5300 of Proceedings of SPIE., San Jose (2004) $1-11$

25. Groen, F.C.A., Verbeek, P.W.: Freeman-coe probabilities of object boundary quantized contours. Computer Graphics and Image Processing 7 (1978) 391-402 
26. Andrès, E.: Defining discrete objects for polygonlization : the standard model. In Braquelaire, A., Lachaud, J.O., Vialard, A., eds.: Discrete Geometry for Computer Imagery. Volume 2301 of Lect. Notes on Comp. Sci., Bordeaux, France, SpringerVerlag (2002) 313-325 